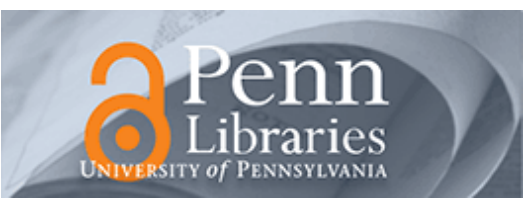

University of Pennsylvania

ScholarlyCommons

Center for Bioethics Papers

Center for Bioethics

September 2003

\title{
Improving Informed Consent: The Medium Is Not the Message
}

\author{
Patricia Agre \\ University of Pennsylvania \\ Frances A. Campbell \\ University of Pennsylvania \\ Barbara D. Goldman \\ University of Pennsylvania \\ Nancy Kass \\ University of Pennsylvania \\ Maria L. Boccia \\ University of Pennsylvania
}

See next page for additional authors

Follow this and additional works at: https://repository.upenn.edu/bioethics_papers

\section{Recommended Citation}

Agre, P., Campbell, F. A., Goldman, B. D., Kass, N., Boccia, M. L., McCullough, L. B., Merz, J. F., Miller, S. M., Mintz, J., Rapkin, B., Sugarman, J., Sorenson, J., \& Wirshing, D. (2003). Improving Informed Consent: The Medium Is Not the Message. Retrieved from https://repository.upenn.edu/bioethics_papers/9

(C) The Hastings Center. Reprinted by Permission. This article originally appeared in IRB: Ethics and Human Research Special Supplement, Volume 25, Issue 5, September 2003, pages S11-S19.

Publisher URL: http://www.thehastingscenter.org/publications/irb/irb.asp

This paper is posted at ScholarlyCommons. https://repository.upenn.edu/bioethics_papers/9

For more information, please contact repository@pobox.upenn.edu. 


\title{
Improving Informed Consent: The Medium Is Not the Message
}

\begin{abstract}
An important type of research on informed consent involves empirically testing interventions designed to improve the consent process. Here we report on the experience of eight teams that conducted research involving interventions designed primarily to impact one of three categories: decision-making, knowledge, and the therapeutic misconception.
\end{abstract}

\section{Comments}

(C) The Hastings Center. Reprinted by Permission. This article originally appeared in IRB: Ethics and Human Research Special Supplement, Volume 25, Issue 5, September 2003, pages S11-S19.

Publisher URL: http://www.thehastingscenter.org/publications/irb/irb.asp

\section{Author(s)}

Patricia Agre, Frances A. Campbell, Barbara D. Goldman, Nancy Kass, Maria L. Boccia, Laurence B. McCullough, Jon F. Merz, Suzanne M. Miller, Jim Mintz, Bruce Rapkin, Jeremy Sugarman, James Sorenson, and Donna Wirshing 


\section{Improving Informed Consent: The Medium Is Not the Message}

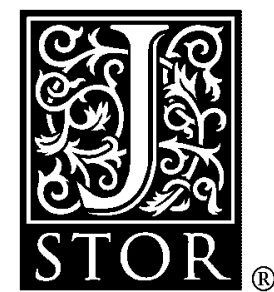

Patricia Agre; Frances A. Campbell; Barbara D. Goldman; Maria L. Boccia; Nancy Kass; Laurence B. McCullough; Jon F. Merz; Suzanne M. Miller; Jim Mintz; Bruce Rapkin; Jeremy Sugarman; James Sorenson; Donna Wirshing

IRB: Ethics and Human Research, Vol. 25, No. 5, Supplement. (Sep. - Oct., 2003), pp. S11-S19.

Stable URL:

http://links.jstor.org/sici?sici=0193-7758\%28200309\%2F10\%2925\%3A5\%3CS11\%3AIICTMI\%3E2.0.CO\%3B2-0

IRB: Ethics and Human Research is currently published by The Hastings Center.

Your use of the JSTOR archive indicates your acceptance of JSTOR's Terms and Conditions of Use, available at http://www.jstor.org/about/terms.html. JSTOR's Terms and Conditions of Use provides, in part, that unless you have obtained prior permission, you may not download an entire issue of a journal or multiple copies of articles, and you may use content in the JSTOR archive only for your personal, non-commercial use.

Please contact the publisher regarding any further use of this work. Publisher contact information may be obtained at http://www.jstor.org/journals/hastings.html.

Each copy of any part of a JSTOR transmission must contain the same copyright notice that appears on the screen or printed page of such transmission.

JSTOR is an independent not-for-profit organization dedicated to and preserving a digital archive of scholarly journals. For more information regarding JSTOR, please contact support@jstor.org. 


\title{
Improving Informed Consent: The Medium Is Not the Message
}

\author{
by Patricia Agre, Frances A. Campbell, Barbara D. Goldman, Maria L. Boccia, Nancy Kass, \\ Laurence B. McCullough, Jon F. Merz, Suzanne M. Miller, Jim Mintz, Bruce Rapkin, \\ Jeremy Sugarman, James Sorenson, and DONNa WirShING
}

$\perp$ $\mathrm{n}$ important type of research on informed consent involves empirically testing interventions designed to improve the consent process. Here we report on the experience of eight teams that conducted research involving interventions designed primarily to impact one of three categories: decisionmaking, knowledge, and the therapeutic misconception. The interventions used included video and computer-based programs to provide information, modified consent documents for low-literacy populations, role-play, and decision-aids. Six of the eight studies were randomized controlled trials; one was a quasiexperimental trial and one involved the development of instruments to assess patient autonomy. Two tested decisional aids, three used computer technology, and three used video.

The outcomes examined included knowledge gained by the study subjects, their satisfaction with the process of informed concent, and/or their decision whether to participate in a clinical trial. Five studies used real patients and three used surrogates. Two of the studies with surrogate patients involved simulated subject recruitment. The subjects in all

Patricia Agre, Frances A. Campbell, Barbara D. Goldman, Maria L. Boccia, Nancy Kass, Laurence B. McCullough, John F. Merz, Suzanne M. Miller, Jim Mintz, Bruce Rapkin, Jeremy Sugarman, James Sorenson, and Donna Wirshing, "Improving Informed Consent: The Medium Is Not the Message," IRB: Ethics \& Human Research Supplement 25 No. 5 (2003): S11-S19. but one study were predominately Caucasian. ${ }^{1}$ Overall, the eight studies comprised relatively well-educated participants. The projects are summarized in Table 1.

\section{Projects on Knowledge Outcomes}

\section{Methods to Improve} Informed Consent Information. Agre and Rapkin compared knowledge of the various elements of consent information among groups randomly assigned to experience one of four methods of presentation: standard consent, video, computer program, or booklet. The three groups were patients deciding whether to participate in a clinical trial, their family/friends, and individuals recruited from the waiting area in a surgical day hospital. The mean age of all study subjects was 53.8 years; $43 \%$ were female and $11 \%$ were ethnic minorities (predominately African-American and Latino). The mean age of patient subjects was 60 years; $26 \%$ were female and $9.6 \%$ were ethnic minorities. The knowledge quizzes included multiple choice questions on each of the elements of informed consent based on the details provided in each specific protocol. They ranged from 12 to 15 questions depending on how involved the research procedure was and the number of side effects.

Significant predictors of correct answers for patient subjects were: (1) protocol complexity (the more com- plex, the more correct answers; $\mathrm{p}<$ $.001)$; (2) education (better educated subjects scored better; $\mathrm{p}<.05$ ); (3) minority status (minorities scored lower; $\mathrm{p}<.05$ ); and (4) social functioning as captured in the Functional Assessment of Cancer Treatment General Form (FACT-G) (patients in greater distress gave fewer correct answers; $\mathrm{p}<.05$ ).

Although main-effect differences in knowledge of patient subjects across the four interventions were not evident, computer and video consent formats produced slight improvements in understanding over booklet and standard versions when protocols involved later phase clinical trials $(\mathrm{p}<.10)$. Video was superior to the computer format for complex protocols $(\mathrm{p}<.04)$ and for minority participants $(\mathrm{p}<.07)$. Patient subjects in poorer health scored better with the booklet format $(\mathrm{p}<.05)$, though the opposite was the case for minority subjects ( $p$ $<.05$ ).

Cross-tabulation analysis examined whether patterns of response to the knowledge quiz were related to the different methods of consent. Responses to the computer and video methods differed considerably from responses to the booklet and standard formats (Chi-square $=$ $18.06, \mathrm{p}<.006)$. In the full sample of 441 subjects, those receiving booklet or standard versions of consent information were more likely to 
Table 1.

Study Summaries

\begin{tabular}{|c|c|c|c|c|c|c|}
\hline Study & Design & Intervention & Measurement & Type & Participants & Outcome \\
\hline Agre et al & $\begin{array}{l}\text { Randomized, } \\
\text { controlled }\end{array}$ & $\begin{array}{l}\text { Print, video, } \\
\text { computer, standard }\end{array}$ & Quiz, FACT-C & $\begin{array}{l}\text { Any clinical } \\
\text { research study }\end{array}$ & $\begin{array}{l}441 \text { ( } 204 \\
\text { patients, } 109 \\
\text { family members, } \\
128 \text { subjects not } \\
\text { involved with a } \\
\text { clinical trial) }\end{array}$ & $\begin{array}{l}\text { Knowledge } \\
\text { (quiz) }\end{array}$ \\
\hline Campbell et al & $\begin{array}{l}\text { Randomized, } \\
\text { controlled }\end{array}$ & $\begin{array}{l}\text { Enhanced print, } \\
\text { videotape, } \\
\text { PowerPoint, } \\
\text { standard }\end{array}$ & $\begin{array}{l}\text { Audiotape of free } \\
\text { recall (scored } \\
\text { for number of } \\
\text { information bits) } \\
\text { and prompted } \\
\text { recall (scored for } \\
\text { correctness and } \\
\text { quality of response) }\end{array}$ & $\begin{array}{l}\text { Parents of Head } \\
\text { Start children } \\
\text { simulating parents } \\
\text { of premature infants } \\
\text { or of children } \\
\text { needing a heart } \\
\text { transplant }\end{array}$ & 233 parents & $\begin{array}{l}\text { Knowledge (free } \\
\text { recall and } \\
\text { prompted } \\
\text { assessments); } \\
\text { willingness to } \\
\text { participate in low } \\
\text { - and high-risk } \\
\text { protocols }\end{array}$ \\
\hline
\end{tabular}

$\begin{array}{lll}\text { Kass et al } & \begin{array}{l}\text { Descriptive followed Computer-based } \\ \text { by randomized, vs. pamphlet } \\ \text { controlled }\end{array} & \begin{array}{l}\text { Audiotape of Phase I, II } \\ \text { consent discussion, cancer } \\ \text { quantitative survey, trials } \\ \text { qualitative in-depth } \\ \text { interview }\end{array}\end{array}$

$87 \quad$ Level of patient understanding of early phase trials

\begin{tabular}{|c|c|c|c|c|c|}
\hline Merz and Sankar & $\begin{array}{l}\text { Randomized, } \\
\text { controlled }\end{array}$ & $\begin{array}{l}\text { Printed consent } \\
\text { form with or } \\
\text { without a short } \\
\text { series of vignettes }\end{array}$ & $\begin{array}{l}\text { Consent for } \\
\text { donation of blood } \\
\text { to DNA bank solely } \\
\text { for research } \\
\text { purposes }\end{array}$ & 206 & $\begin{array}{l}\text { Knowledge } \\
\text { (survey) }\end{array}$ \\
\hline
\end{tabular}

\begin{tabular}{lllll}
\hline Miller et al & $\begin{array}{l}\text { Randomized, } \\
\text { controlled }\end{array}$ & $\begin{array}{l}\text { Enhanced } \\
\text { counseling vs. } \\
\text { standard counseling } \\
\text { with general health } \\
\text { information control }\end{array}$ & $\begin{array}{l}\text { BRCA genetic } \\
\text { testing }\end{array}$ & $\begin{array}{l}\text { 276 } \\
\text { womal style }\end{array}$ \\
& & & BRC
\end{tabular}

\begin{tabular}{lll}
\hline Mintz et al & $\begin{array}{l}\text { Randomized, } \\
\text { controlled }\end{array}$ & Videotape \\
\hline Sorenson & $\begin{array}{l}\text { Quasi-experimental } \\
\text { design }\end{array}$ & $\begin{array}{l}\text { Decision-aid } \\
\text { vs. standard }\end{array}$
\end{tabular}

Measurement development

$\begin{array}{lll}\text { Autonomy } & \text { Cooperating } & 170, \text { including } \\ \text { instrument; barriers } & \text { randomized, } & \text { geriatric patients } \\ \text { to autonomy } & \text { controlled clinical } & \text { with chronic illness } \\ \text { instrument } & \text { trials } & \text { and psychiatry } \\ & & \text { patients }\end{array}$

Women at risk for 139

hemophilia A

carrier status instrument; barriers to autonomy
276 Satisfaction,

women at risk for risk-related,

BRCA carrier status intrusive

/ avoidant ideation, intentions to communicate test results to family

Knowledge (pre/post quiz)

User-friendliness of decision-aid, differences in accepting testing, harms and benefits identified by subjects

McCullough $\quad \begin{aligned} & \text { Measurement } \\ & \text { development }\end{aligned}$
patients

\section{Validation analysis to be completed will result in first comprehensive measure of autonomous decisionmaking for enrollment in Phase III clinical trials}


demonstrate specific errors, such as misunderstanding privacy, compensation, or alternative interventions. Very similar findings were evident in analyses including patient subjects only.

\section{Improving the Consent} Process for Parents. Campbell, Goldman, and Boccia conducted a simulated recruitment using the consent forms from two actual pediatric studies (one high-risk, one low risk). Parents of children in a Head Start program were recruited for the purpose of the consent study; they were not participants in the actual protocols involving children. The study was designed to determine whether modifying the presentation format of consent information would affect knowledge gained and stated willingness to enroll their children in the sample pediatric studies. Across all presentation methods, knowledge gained was assessed with free and prompted recall.

Four versions of consent forms were compared: the unchanged original print version; a modified print version formatted to enhance readability with increased white space, bold headings, and the addition of photographs and figures; a narrated videotape; and a PowerPoint version that permitted participants to selfpace through bulleted text on-screen with accompanying photographs and narration. All versions contained essentially the same amount of information. To control for participant reading ability, the level of reading comprehension was assessed with the Woodcock-Johnson PsychoEducational Battery, Part 2. ${ }^{2}$ The four consent tools were tested with and without using reading comprehension scores as a covariate.

Approximately $25 \%$ of the parent subjects had less than a high school education; $49 \%$ had at least some college or vocational training. Eighty-five percent were women and $84 \%$ were African American. Reading comprehension raw scores ranged from 11 to 40 , with a mean score of 28.1 , reflecting a reading grade equivalent of 8.1 , with a standard deviation of 5.1 grades. Outcomes were examined for the entire sample, and for a subsample that tested at or below the 8th grade level in reading comprehension.

In the entire sample, with reading level covaried, none of the modifications made to the original consent forms resulted in significant improvements in either free or prompted recall. Parent subjects appeared to be sensitive to the risk entailed by the study in which they were asked to consider enrolling their child. Significantly more of them indicated that they would not enroll a child in the high-risk protocol. Thirty-four percent indicated refusal compared to $8.5 \%$ in the low risk protocol $(\mathrm{p}=.007$ for the $\mathrm{Chi}$ Square test). In the subsample of poorer readers $(n=124$ or $53 \%$ of the total sample), there was a trend for the modified text version to be superior to the original $(\mathrm{p}=.055)$ and video versions $(\mathrm{p}=.066)$, suggesting that textual modification may be important for those with poorer reading skills, but less relevant for those with good skills. However, though all the modified versions were based on recommendations from past research on ways to enhance comprehension, the study results were not as dramatic as the investigators had expected.

\section{- Improving Patient} Understanding of Early Phase Oncology Trials. The study by Kass, Sugarman, Taylor, and Fogarty had three goals: (1) to assess the level of understanding of the purpose, risks, and benefits of early phase clinical trials (EPCTs) among oncology patients offered enrollment in a phase I or phase II clinical trial;

(2) to create an intervention designed to increase cancer patients' understanding of early phase clinical trials; and (3) to assess the effect of the intervention on patient understanding and the nature of physicianpatient discussions.
This project was conducted in two stages. The first was a descriptive study that included audiotaping 77 patient appointments during which oncologists invited patients to participate in EPCTs; patients were interviewed afterwards. The mean age of patient subjects was 62 years; $2 / 3$ were male, $85 \%$ were Caucasian, and almost half had at least a college degree. Approximately $80 \%$ decided to enroll in a clinical trial with the rest choosing standard treatment. Fifty-eight percent of patient subjects believed the purpose of the oncology trial was to "see if the drug works," $16 \%$ said the purpose was "to see if the drug helps me," and $9 \%$ said the purpose was to "cure my cancer." By taking part in the trial, $55 \%$ said they expected to experience some long-term improvement in their condition or a complete cure of the cancer.

Audiotapes of medical encounters in which physicians introduced the possibility of participation in an EPCT and its alternatives (the "options discussion"), revealed that physicians often gave patients mixed messages. Physicians often were quite candid that the main purpose of the trial was to determine the right drug dosage. In some cases they also stated explicitly that the drug had not been previously used in humans, or acknowledged that good ideas in cancer treatment often do not pan out. Yet in almost the same breath, physicians typically told patients they would be kept on the experimental drug "as long as it worked," or they asked patients if they were risk takers, willing "to try to go for that chance." Comments such as these lend support to patients' beliefs that the trial actually might help them clinically, and was being offered for that purpose.

Analysis of the options discussions compared to routine medical appointments showed that physicians provided more information about treatment, third parties were more active in discussions, physi- 
cians stated worry and concern and provided reassurance and optimism more than average, and they were rated higher on interest/attentiveness, friendliness/warmth,

responsiveness/engagement, and sympathy/empathy.

In response to these results, the project team developed a narrated 2o-minute, self-paced, touch screen computer-based intervention that described the nature of clinical trials, provided sample questions for patients to ask their oncologists, and included video clips of patients and oncologists describing EPCTs.

Patients eligible for EPCTs were randomly assigned to the computerbased intervention or to receive a pamphlet about cancer clinical trials developed by the National Cancer Institute (NCI).

Data have been analyzed from the first 87 patient subjects ( 46 from the pamphlet group of the projected goal of 100 patient subjects). Patient subjects in both groups were equally likely to decide to join an EPCT, though those in the computer group were significantly more likely to say the information changed the way they made their decision than those in the pamphlet group $(27 \%$ vs. $7 \%$, $\mathrm{p}=.004)$. Both groups of subjects said that the computer and pamphlet formats were informative, helpful, and clearly presented, and indicated they would recommend these formats to other patients. Patient subjects in the computer group found this tool easy to use, and reported the video clips of patients as the most useful part of the computer intervention. However, patient subjects in the pamphlet group were more likely to report that they expected to stick with their decision (39\% vs. $20 \%, p=.05)$ whether to participate in an EPCT.

Informed Consent to DNA Banking for Research. Merz and Sankar focused on the development of an informed consent form and process for donation of blood to a DNA Bank to be used solely for research purposes. Study subjects were recruited from the General Clinical Research Center (GCRC) or the apheresis unit at the Hospital of the University of Pennsylvania. They were asked to pretend they had been asked to donate blood for research purposes. Subjects were randomly assigned to receive the consent form or the consent form plus a short series of vignettes designed to spur conversation as well as to prompt cognitive processing of the information in the consent form. After the informed consent discussion, subjects were given a survey instrument with questions about their knowledge and opinions of the consent form, their perceptions and beliefs about risk and benefit, and their decision whether or not to donate blood. They were also asked to provide basic demographic information.

Of 255 individuals who were approached, 206 completed the consent form, but only 197 also completed the demographic information. Subjects were evenly divided by sex, were predominantly Caucasian (159), and were relatively well educated $(48 \%$ had at least some college, $22 \%$ had graduate degrees). Twenty-five percent of the subjects asked questions during the consent process. Exploratory multiple logistic regression showed that subjects more likely to ask questions were those who found the form harder to read $(\mathrm{OR}=1.3, \mathrm{p}=0.006)$ and those who believed they were at higher personal risk from the research (OR $=1.2, p=0.02)$. Subjects who had medical training or work experience were less likely to ask questions (OR $=0.13, p=0.009$ ).

Subjects demonstrated relatively good understanding of the information in the consent form, completing on average 17 of 21 post-presentation questions correctly. The vignettes had no significant effect (min $\mathrm{p}>0.45$ ) on number of correct responses. Multiple linear regression showed that education was the strongest predictor of correct responses, with those having college or more education getting on average 1.4 more questions correct $(\mathrm{p}<$ 0.001 ). Additional findings revealed that those with medical education or experience got 1.3 more questions correct $(p=0.006)$, subjects in the apheresis unit got 0.8 fewer questions correct than subjects in the GCRC, and those who identified themselves as other than Caucasian got 1.4 fewer questions correct $(\mathrm{p}=$ 0.004 ).

Consenting to Medical and Psychiatric Treatment Research. Mintz, Wirshing, and colleagues produced two videos. One video ("experimental") was designed explicitly to prepare patients for participating in the informed consent process for medical and psychiatric treatment research. It explains the major points federal regulations require to be included in consent forms and outlines the elements of good decisionmaking. Using a combination of dramatized vignettes, voice-over, and bulleted text, the video encourages patients to adopt an active participation style during the consent session. The second video ("control") was designed to be appropriate for use in studies of consent, but contains no modeling or teaching specific to the consent process. It discusses the need for human subject protections in general, presents some historical material on the topic, and describes the federal regulatory apparatus including institutional review boards (IRBs) and their role.

This project has recruited subjects with schizophrenia (plus a small group with bipolar disorder) who are in the process of considering participation in ongoing treatment research at one of several participating venues at the West Los Angeles Veteran's Affairs (VA) Medical Center and the University of California, Los Angeles. The videos have also been evaluated in comparison samples of medical patients at the West Los Angeles VA Medical 
Center and of college students.

Patients were recruited for the project after they were identified as potential participants in a cooperating treatment study, but before any actual recruitment for the treatment study had occurred. Prior to the treatment research consent session, they were asked to volunteer for the consent project. They met with the staff of the informed consent study, completed assessments of psychological and social history and decisionmaking, watched one of the two videos selected at random, and took brief pre- and post-quizzes on the video content. Subjects then went to the treatment research study team to participate in the usual treatment study consent session. The treatment study recruiter completed a brief checklist following the session that described the participant's behavior. In two subsequent sessions, subjects were assessed to determine their understanding and memory for the details of the treatment research study, and completed a battery of neurocognitive tests and psychodiagnostic assessments. A session three months later determined memory for study details, understanding of the treatment research study, and the decisionmaking process. A group of medical patients awaiting appointments (not involved in treatment research) served as a medical (not mentally ill) control group.

Preliminary data analyses have been performed for the schizophrenia and medical groups. Seventy eight patient subjects with schizophrenia have viewed the videos (43 viewed the experimental video). Eighty-one percent were male, with a mean age of 38.14 years (range 18 64). Twenty-nine percent were Caucasian, $31 \%$ African American, $9 \%$ Latino, $10 \%$ Asian, and $21 \%$ other. Eight percent had less than a high school degree, $41 \%$ had a high school degree, $37 \%$ some post-high school education, and $14 \%$ had 16 or more years of education. Sixtyseven percent had prior research par- ticipation. The median number of psychiatric hospitalizations was 4 .

In the medical patient sample, 83 patient subjects (some with selfreported psychiatric co-morbidities) viewed the experimental teaching video, and 39 viewed the control video. Eighty-nine percent of these participants were male, with a mean age of 56.12 (range 25-87). Median education level was 13.8 years (range 9-18). Six percent had less than a high school degree, $20 \%$ had a high school degree, $48 \%$ had some post-high school education, and $26 \%$ had 16 or more years of education.

Patient subjects with schizophrenia who viewed the experimental video improved more in knowledge of the consent process than those viewing the control video $(\mathrm{t} 4.01, \mathrm{df}$ $69.5,0.0002$ ). A similar result was obtained among medical patients $(\mathrm{t}$ 3.88 , df 120, 0002). Those viewing the experimental video showed greater increases in knowledge of the informed consent process than those viewing the control video, whether they reported co-morbid psychiatric illness $(\mathrm{t}=2.5, \mathrm{df}=58, \mathrm{p}=.02)$ or not $(t=3.07, \mathrm{df}=58.8, \mathrm{p} .=003)$. Analyses of the neuropsychological assessments, measures of competen$\mathrm{cy}$, and decisionmaking are in progress.

\section{Projects on Decisionmaking}

\section{Facilitating Well-Informed} Decisions for BRCA Testing. Miller et al. developed and evaluated a theoretically-guided enhanced counseling protocol, Cognitive-Affective Processing (CAP), to facilitate wellinformed decision making about $B R C A$ genetic testing among women with family pedigrees suggestive of high risk for breast cancer. ${ }^{3}$ The CAP intervention is conceptually based on the Cognitive-Social Health Information Processing (C-SHIP) model, which provides a unifying framework for specifying the principles needed to guide the application of behavioral science to the design of genetic screening and testing counseling interventions.

According to the C-SHIP model, individuals generally display stable cognitive-affective patterns, or psychological signatures, in how they process information about threatening medical feedback. ${ }^{4}$ In particular, individuals can be characterized as high monitors (who attend to, scan for, and exaggerate health threats) and low monitors (who distract from, ignore, and downplay health threats). However, people are often unaware of their own likely pattern of response, particularly when the threat is unfamiliar or uncertain, as in the case with genetic testing. The goal of the CAP procedure is to help individuals realistically to anticipate and plan for the personal consequences of learning their carrier status, thereby facilitating informed decisionmaking and adjustment over time. The CAP procedure systematically enables genetic testing candidates vividly to anticipate and enact in specific detail, through structured role-play protocols, the receipt of both positive and negative/indeterminate genetic test results and their potential responses to them.

Participants in the study underwent standard education and counseling, and were then randomly assigned to the CAP session or to a General Health Information (GHI) control session (to equate for time and attention). They were subsequently given the option of donating blood for BRCA testing.

Psychosocial assessments were obtained five times: before the educational session (baseline), before the individualized enhanced or standard counseling intervention, one week post-intervention (after blood draw), before the predisclosure session, and before the disclosure session. Outcomes of interest included riskrelated distress (i.e., intrusive and avoidant ideation), knowledge, satisfaction with the genetic testing decision, and intentions to communicate test results to family members. 
Participants ( $\mathrm{n}=276)$ were primarily Caucasian $(95 \%)$, unaffected by breast and ovarian cancer $(61 \%)$, and well educated $(47 \%$ with undergraduate degrees or higher). As expected with this at-risk sample, $93 \%$ percent chose to donate blood for testing, and baseline levels of knowledge were generally high.

Interim analyses conducted to date on a subset of women $(n=100)$ revealed that CAP participants had significantly higher levels of riskrelated intrusive ideation (but not more avoidant ideation) 1 -week post-intervention than GHI participants, suggesting that they were more engaged in the cognitive-emotional processing of testing participation $(\mathrm{p}<.01)$. In addition, there was a significant main effect for the type of counseling session (CAP vs. GHI) regarding satisfaction with the genetic testing decision $(\mathrm{p}<.05)$. Women who received CAP, and thereby were able to more thoroughly and deeply process their complex relations to threat, were more satisfied than women who received the GHI. Finally, women receiving the CAP intervention demonstrated a greater increase in their intention to seek advice about how to communicate positive $B R C A \quad 1 / B R A C 2$ test results effectively to family members, compared with women receiving the GHI $(\mathrm{p}<.04)$. Additional monitoring was also associated with a greater increase in intention to communicate test results to family members, regardless of the test outcome (i.e., positive vs. negative; $\mathrm{p}<05$ ).

An Experimental Study to Improve Risk/Benefit Appraisal. Sorenson et al. developed, implemented, and assessed the effectiveness of a theoretically based decision-aid for use by potential research participants during the informed consent process. The decision-aid was designed to assist at-risk women in deciding whether to participate in a research project offering free hemophilia A carrier testing. The decision-aid, based on Janis and
Mann's Conflict Theory of Decision Making, ${ }^{5}$ was a four-page paper and pencil instrument. It asked women to consider the consequences of accepting or declining hemophilia A carrier testing for themselves and for their significant others.

A total of 139 women were enrolled in the study. Sixty-one received the decision-aid as part of the informed consent process and 78 received standard consent information only. Just over half the women had a high school education or less, nearly $80 \%$ were non-Hispanic Caucasian, $75 \%$ were Protestant, and just under $70 \%$ were married. Three-fourths had completed their childbearing and two-thirds had never undergone non-DNA based carrier testing. During a baseline telephone interview, information was collected on past experiences with hemophilia A and on knowledge of both carrier testing and of hemophilia. Six months later, the women completed a second telephone interview covering most of these same topics.

The research focused on four questions: (1) Could a decision aid be incorporated into the consent process? (2) Could women of varying educational backgrounds complete the decision-aid? (3) Would completion of the decision-aid lead to differential rates of accepting/declining hemophilia $\mathrm{A}$ carrier testing? and (4) What harms and benefits would women list as most important in their decisionmaking?

The decision-aid did not disrupt the consent process. Moreover, women of varying educational levels successfully completed the decisionaid. There was no statistically significant difference in the rates of accepting/declining carrier testing between the treatment $(80.3 \%)$ and control groups $(83.3 \%)$. Finally, women identified a broad spectrum of reasons they had considered in making their decision, with concerns about the impact of accepting or declining testing on immediate relatives being a significant consideration. These considerations were in marked contrast to those identified by the researchers in the standard consent form, which focused on potential discrimination, needle infection, and the personal value of knowing one's own carrier status.

Enhancing the Autonomy of Vulnerable Individuals. The overall goal of the project by McCullough and colleagues is to enhance the autonomy of vulnerable individuals by reducing barriers to their exercise of autonomy in the informed consent process. As a first step, the study team developed instruments to measure the frequency of barriers to the exercise of autonomy in the consent process for research and to measure the exercise of autonomy comprehensively. Autonomy is viewed as having multiple dimensions, including intentionality, understanding, and non-control. ${ }^{6}$ The autonomy questionnaire begins with some general questions about the consent and using information (that operationalize the concept of intentionality), a section on control of the decisionmaking process and the decision to sign or not sign the consent form (to operationalize voluntariness), and a section on understanding the study, including items concerning a study's benefits, risks, alternatives, and confidentiality. The barriers questionnaire contains items drawn from the literature and focusgroup research on a wide variety of aspects of the consent process that could be experienced by subjects as barriers to the exercise of autonomy. Data collection for the validation of both instruments has been completed and analysis is underway. The two instruments should be ready for use by other investigators in the near future. This work is seen as a challenge to the "protective approach" to research with vulnerable subjects that responds to vulnerability by either excluding subjects from participation in research or by adding 
requirements to the informed consent process. ${ }^{7}$ As their next step of research, McCullough and colleagues intend to explore changes in the consent process that would increase the autonomy of vulnerable subjects, so that they can consent for themselves, obviating the need for the protective approach.

\section{Overall Effects Across Intervention Studies}

To our surprise, most of the interventions did not significantly improve knowledge of consent information. The most powerful intervention involved video-based preparation for patient subjects prior to encountering the consent process for medical or psychiatric treatment research. ${ }^{8}$ In that project, quizzes given before and after the intervention indicated that knowledge of consent information and the consent process were significantly improved. In contrast, average percent correct on knowledge quizzes, regardless of intervention, was $80 \%$ in the Merz and Sankar study, about $69 \%$ in the Agre et al. study, and about $50 \%$ in the Campbell et al. study. However, the assessment of knowledge occurred as a post-test after the intervention and across presentation methods, rather than contrasting before and after (pre-test/post-test), within subjects. A majority of patient subjects in the Kass et al. study involving EPCTs incorrectly described therapeutic aims as a purpose of the trial, even after viewing the intervention video.

No single alternative method of providing consent information was clearly superior for all subjects and target groups in the studies we examined. Project teams that evaluated video interventions agreed that the cost of these consent tools may outweigh their benefit given the dearth of improved outcomes. 9 Computer-based information systems were slightly better than print consent formats in some (poorer readers), but not all instances. ${ }^{10}$
Modified print slightly improved knowledge in some, ${ }^{11}$ but not in all studies, ${ }^{12}$ even though the print material contained recommended practices-plenty of white space, headers, adequate font size, and reading level adjusted for the target audience.

Information given to patients in four of the studies varied, and certain types of information made some patients uncomfortable. ${ }^{13}$

Information that facilitated the anticipation of cognitive and emotional consequences of participation in genetic testing appears to have increased both knowledge and the number of intrusive ideas women experienced when contemplating test results, but not the number of avoidant ideas, suggesting that more active and accurate threat processing had occurred. ${ }^{14}$ In another context, vignettes did not increase recall of information, but this group of subjects had a relatively good understanding of the consent form, on average answering 17 of 21 questions correctly. ${ }^{15}$ Explicitly preparing patients for active participation versus simply informing them of IRB procedures in general significantly improved knowledge of the consent process and details of the subsequent research. ${ }^{16}$

Although decision-aids and roleplay did not change decisions to take part in a study, they did result in other outcomes of interest. Both surrogate and authentic subjects in all groups were highly likely to take part in the research protocol. Their decision to participate was not impacted by the intervention, suggesting that subjects might make their decisions before receiving the actual informed consent information. ${ }^{17}$ The decision-aid generated a much longer list of motivators than those suggested by researchers.

The role-play impact of the CAP intervention resulted in greater satisfaction for high monitors one-week post-intervention, at the same time as it increased intrusive ideation (from low to moderate) and knowledge, compared to those receiving the GHI intervention. Intentions to share genetic testing results also changed through the course of the testing. In particular, women who received the CAP intervention were more likely to seek professional advice for communicating positive results to family members. These results indicate that women may benefit from an informed consent process that enables them to anticipate, and prepare for, the potential challenges that arise for them personally as a result of testing. Further, the finding that high and low monitors reacted in distinctive patterns to standard vs. enhanced counseling indicates the need to tailor the informed consent process to individual differences, such as attentional style.

Informed consent statements tend to rely largely on professional identification of the potential harms/risks and benefits of research participation, with the list of risks and benefits focusing on the more immediate and personal consequences of participation. Data from the Sorenson study suggest that, at least for genetic carrier testing, potential research participants consider a broad array of issues, including not just personal harms and benefits, but their values, beliefs, motives, and the consequences of their participation on selected significant others. Relying on experts to construct the risk/benefit component of informed consent statements may be limiting the decision utility of consent statements.

The informed consent projects identified a number of attributes that consistently predicted increased knowledge of consent form information: younger age, higher education, better reading ability, and medical background. In one study, knowledge was better if the consent was more complex, perhaps suggesting a greater attentiveness to risk. ${ }^{18}$ In two studies, minority status predicted lower knowledge scores, ${ }^{19}$ yet 
because minority subjects represented a very low percentage of the total samples, this finding may not be valid.

\section{Lessons Learned}

WJe learned valuable lessons in our research on ways to improve the informed consent process. A major concern was that examining the consent process itself would have a negative impact on subject recruitment into clinical trials. It was therefore of interest to find that the informed consent studies did not reflect any change in accrual rates. In addition, none of the project teams reported an unusual rate of refusals to participate in the informed consent research. Patients and non-patient subjects alike were willing to become involved. However, some of the project teams experienced barriers to conducting this type of research. ${ }^{20}$

We also recognized that the control arms in all studies ensured that current best standard practice was realized and that many studies offered enhanced interventions to the control arm participants (one-on-one counseling, booklets that might not routinely be distributed, quizzing with feedback, etc.). Thus, it may have been more difficult to see clear differences between the intervention and control arms than if a more realistic representation of true current practice had been used for comparison.

Motivations for taking part in some clinical research may not be what researchers assume. In the case of genetic testing for hemophilia A carrier status, subjects identified personal reasons for wanting the testing. This suggests that consent forms should be piloted with a sample of the target population so that the risk/benefit ratio includes factors that are important to potential participants. Given the variation in motivation, at least in certain contexts, such as genetic screening, risk/benefit information should be presented in a value neutral manner (e.g., "Some people have experienced ..."). This approach counters the assumption that certain factors investigators believe are negative will be perceived as such by all subjects.

Many investigators agree that written consent forms should include adequate white space, headers, and graphics. This probably should be the standard format, though data from the studies we reviewed are not sufficiently strong to justify mandating such an approach. Modifying printed forms was certainly the least expensive of all the format changes evaluated in these studies and none of the others was clearly better. Modifying print is a low-tech approach accessible to all.

\section{Limitations}

$M$ any of the investigators tested a number of variables simultaneously, making it more difficult to separate the medium from the message. Some studies used hypothetical situations, making it difficult to know whether subjects who are asked to imagine themselves in a particular situation actually duplicate the experience of real patients in real decisions. Finally, most of the studies were limited to one or a small number of contexts. The small effects found here, both positive and negative, will have to be retested in broader situations.

\section{Future Directions}

$\mathrm{I}_{\mathrm{m}}^{\mathrm{t}}$ was clear that many individuals made their decision to take part in a trial before the consent process occurred. We believe that it is important to learn more about when and how individuals make decisions about enrolling in studies so that interventions are more appropriately timed in relation to the decisionmaking process.

Another promising avenue is to investigate how peoples' beliefs affect their understanding of a particular study. Adult learning theory suggests that individuals come to any learning situation within the context of their particular life experiences. The $\mathrm{NCI}$ is attempting to address overall understanding about clinical research with a major educational effort comprising booklets and videos for the public, potential subjects, and trainers of those enrolling subjects. ${ }^{21}$ We may see better outcomes as this effort becomes fully implemented.

Although anxiety did not appear to play a large role in any findings, one study did find a slightly increased risk of a particular type of threat-related distress, as opposed to general anxiety. ${ }^{22}$ The role of affect in the context of informed consent should be further addressed. For example, are there ways to determine whether an anxious patient might make a decision he/she would later regret, or whether certain types of anxiety facilitate decisionmaking and adjustment, particularly over the long-term? Do potential research participants process additional information differently when they are more anxiously focused on threat compared to when they are not?

A further question is what is or should be the "gold standard" of understanding? What should we expect people to know? Many of the intervention studies used knowledge quizzes to assess understanding, but what would qualify as adequate performance on such a quiz? Do we expect subjects to answer $70 \%$ of the questions correctly, $80 \%$, or $100 \%$ ? Do scores on knowledge quizzes actually reflect an understanding of the information provided? Are all of the elements of equal importance, and if not, which are most important? Is it more critical to know a study's purpose or the potential side effects of the experimental intervention? Do we expect subjects to recognize all of the (often) large numbers of side effects? These questions have not been well addressed in the literature, and the studies described here do not adequately address them. 
Some of the studies used generic interventions rather than study-specific ones; this is another area that deserves comparative study. Several of the projects used techniques to encourage participants to identify their own motivations and concerns. We believe that steering informed consent discussions towards prospective subjects' concerns is a promising direction. However, it remains to be seen how this could be accomplished without first giving individuals enough information about a trial to identify what their concerns might be.

The issue of shortened or simpler consent documents was not thoroughly examined in any of the intervention studies. This area requires additional research.

An interesting approach that would address concerns about variation in learning would be to offer potential subjects a choice of the format that delivers information. ${ }^{23}$ Although this might be impractical in a real situation, a standard of the Joint Commission on Accreditation of Healthcare Organizations is that all patients should be assessed for preferred learning style. ${ }^{24}$ Another approach worth investigating would be to assess a potential subject's readiness to make decisions and create interventions for researchers to help potential subjects focus on the process. This approach meets potential subjects at their own stage of readiness. Research that does not simply repackage the same information, but instead focuses on techniques to process information, or to assess subjects' prior beliefs, would be very helpful. Motivation and prior beliefs may skew willingness or ability to listen to information, thereby skewing understanding.

A clearer understanding of the dimensions of autonomy in the consent process and of the barriers to the exercise of autonomy is needed. ${ }^{25}$ Such information would enhance our understanding of how personal characteristics and individ- ual differences of potential research participants affect our ability to treat these persons with the utmost respect for their rights and needs.

\section{Conclusion}

Tone of the intervention studies 1 clearly identified significantly better methods to increase knowledge gained from the consent process, to enhance satisfaction with decisions, or to affect actual decisions. However, many of us found trends suggesting improvements in certain features related to understanding, knowledge, and/or satisfaction. The fact that several studies had fairly powerful control arms could have reduced our ability to detect significant effects attributable to our specific manipulations. In aggregate, the findings support further research into some forms of media, especially the use of computer-based interactive programs and the use of modified or enhanced print in written forms, though we acknowledge that the results seen here do not show conclusively that these interventions improve the informed consent process. A rethinking of how we facilitate information processing may allow better use of media consent tools. Finally, the preponderance of no significant results suggests that we may need to modify our analytic approach. Manipulating the communication technique may be less important than looking more carefully at information processes.

\section{References}

1. Campbell, Goldman, and Boccia.

2. Woodcock RW, Johnson MB.

Woodcock-Johnson Psycho-Educational Battery Revised. Allen, TX: DLN, 1989. 3. Miller SM, Fang CY, Manne SL, et al. Decision making about prophylactic oophorectomy among at-risk women: Psychological influences and implications. Gynecologic Oncology 1999;75: 406-412. 4. Miller SM. Monitoring versus blunting styles of coping with cancer influence the information patients want and need about their disease. Cancer 1995;76: 167-177. 5. Janis I, Mann L. Decision-Making: A Psychological Analysis of Conflict, Choice, and Commitment. New York: The Free Press,
1997.

6. Faden RR, Beauchamp TL. A History and Theory of Informed Consent. New York: Oxford University Press, 1986.

7. Brody BA. Research on the vulnerable sick. In: Kahn JP, Mastroianni AC, Sugarman J. eds., Beyond Consent: Seeking Justice in Research. New York: Oxford University Press, 1998.

8. Mintz and Wirshing.

9. Agre P, Rapkin B, Dougherty J, Wilson R. Barriers encountered conducting informed consent research. IRB: Ethics \& Human

Research 2002;24(4): 1-5; Campbell, et al.; Kass et al.

10. Campbell et al.

11. Agre et al.

12. Campbell et al.

13. Kass et al.

14. Miller et al.

15. Merz and Sankar.

16. Mintz et al.

17. Miller et al.

18. Agre et al.

19. Merz and Sankar; Agre et al.

20. See ref. 9, Agre et al. 2002; Sachs G, Hougham GW, Sugarman J et al. Conducting empirical research on informed consent: Challenges and questions. IRB: Ethics o Human Research Supplement 2003;25(5): $\mathrm{S}_{26}-\mathrm{S}_{32}$.

21. See http://www.cancer.gov/clinicaltrials/resources/clinical-trials-education-series. 22. Miller et al.

23. Skinner CS, Schildkraut JM, Berry D, et al. Pre-counseling education materials for BRCA testing: Does tailoring make a difference? Genetic Testing, in press.

24. Joint Commission on Accreditation of Healthcare Organizations. Joint Commisssion, Comprehensive Accreditation Manual for Hospitals, The Official Handbook. Chicago:

Joint Commission on Accreditation of

Healthcare Organizations, 2002. 25. See ref. 7, Brody 1998. 